\title{
Microarray expression profile analysis of long non-coding RNAs in optineurin E50K mutant transgenic mice
}

\author{
YUANYUAN LI, LIN JIN, AIMENG DONG, XINRONG ZHOU and HUIPING YUAN \\ Department of Ophthalmology, The Second Affiliated Hospital of Harbin \\ Medical University, Harbin, Heilongjiang 150081, P.R. China
}

Received April 12, 2016; Accepted March 23, 2017

DOI: $10.3892 / \mathrm{mmr} .2017 .6722$

\begin{abstract}
The biological role of long non-coding RNAs (lncRNAs) involves various cellular processes and leads to human diseases. Mutations in the optineurin (OPTN) gene, including E50K, which encodes an amino acid substitution, have been associated with primary open angle glaucoma (POAG). The present study was designed to identify lncRNAs associated with OPTN (E50K) transgenic mice and investigate its functions in the pathogenesis of POAG. The retinas from six OPTN (E50K) transgenic and wild-type mice were collected separately, and lncRNA expression profiling was performed using microarray analysis. Based on Pearson's correlation analysis, an lncRNA and mRNA co-expression network was constructed. Gene Ontology (GO) and Kyoto Encyclopedia of Genes and Genomes enrichment analysis of the lncRNAs and coexpressed mRNAs was used to identify the associated biological modules and pathological pathways. The GO biological processes (BPs) of the differentially expressed lncRNAs were predicted using a computational method of gene set enrichment analysis. A total of 69 IncRNAs showed differential expression between the OPTN (E50K) transgenic mice and wild-type mice, which included 37 downregulated and 32 upregulated lncRNAs. The pathway analysis revealed that the lncRNAs coexpressed with mRNAs were enriched in mRNA surveillance and RNA transport pathways. In addition, eight lncRNAs were annotated in the GO BPs, and two of these eight lncRNAs, ASMM10P055228 and ASMM10P040128, were annotated with the negative regulation of oxidative stress-induced cell death and regulation of execution phase of apoptosis. These results showed that IncRNAs were differentially expressed in the retinas between OPTN (E50K) transgenic and wild-type mice, and
\end{abstract}

Correspondence to: Dr Huiping Yuan, Department of Ophthalmology, The Second Affiliated Hospital of Harbin Medical University, 246 Xuefu Road, Nangang, Harbin, Heilongjiang 150081, P.R. China

E-mail: yuanhp2013@126.com

Key words: optineurin (E50K), long non-coding RNA, mRNA, microarray analysis this may be important in the pathogenesis of POAG caused by the OPTN (E50K) mutation.

\section{Introduction}

Glaucoma is a neurodegenerative ocular disease, which is recognized worldwide as the key causal factor in irreversible blindness (1). The predominant form of glaucoma is primary open-angle glaucoma (POAG). There are multiple genetic factors, which are significant in the etiology of glaucoma. There are $>20$ genetic loci associated with POAG, however, only a few of these have been identified, including myocilin, WD-repeat domain 36, optineurin (OPTN), and TANK-binding kinase-1 (2,3). The OPTN gene mutations, which encode amino acid substitutions including R545Q, E50K and H486R, have been linked to POAG and normal-tension glaucoma (NTG) (4-7). In a previous study, it was shown that, in transgenic mice models, the OPTN (E50K) mutation was the key element leading to the apoptosis of retinal ganglion cells, however, the specific mechanism remains to be fully elucidated (8).

Long noncoding RNAs (lncRNAs) are defined as structurally resembling mRNAs and transcribing $>200$ nucleotides, but not encoding proteins. These IncRNAs have been considered to be transcriptional noise; however, increasing evidence suggests that lncRNAs are important regulators governing various biological processes (BPs), including genomic imprinting, transcription activation and inhibition, chromatin modification, and tissue development (9). POAG is a complicated pathological process, which mediates the gene regulatory network. The latent function of lncRNAs in the retina of OPTN (E50K) transgenic mice remains to be elucidated. The present study analyzed the expression profile of differentially expressed lncRNAs in OPTN (E50K) transgenic and wild-type mice. It was found that eight lncRNAs were annotated in the GO BPs, and two of these eight lncRNAs, ASMM10P055228 and ASMM10P040128, were annotated with negative regulation of oxidative stress-induced cell death and regulation of execution phase of apoptosis, which may be the underlying mechanism for POAG.

\section{Materials and methods}

Animals and sample collection. All animal experiments were performed according to the guidelines of the National Institutes 
of Health and Regulations on the Care and Use of Laboratory Animals (National Institutes of Health, Bethesda, MA, USA). The retinas from 8-month-old transgenic and wild-type mice were collected. The retinas from six transgenic and wild-type mice were divided into three groups (E1, E2 and E3) and (W1, W2 and W3), respectively and were analyzed independently for the expression of lncRNAs.

RNA extraction. Total RNA from each sample was extracted using TRIzol reagent, and quantified using a NanoDrop ND-1000 spectrophotometer; and RNA integrity was evaluated using standard denaturing agarose gel electrophoresis.

Microarray analysis. The expression profiles of the genome-wide mRNA and lncRNAs of the mice were examined using Arraystar mouse lncRNA microarray version 3.0, designed for the profiling of mouse genome-wide lncRNAs and protein-coding transcripts, performed by KangChen Biotech Co., Ltd. (Shanghai, China). This microarray contains 35,923 lncRNAs collected from the National Center for Biotechnology Information Refseq, UCSC, Ensembl, RNAdb 2.0, Fantom3, ncRNA expression databases, and from previous literature. A total of 24,881 coding transcripts were extracted based on the Collaborative Consensus Coding Sequence (CCDS) public source.

The microarrays were performed using an Agilent scanner (G2505C; Agilent DNA microarray scanner; Agilent Technologies, Inc., Santa Clara, CA, USA) and the captured array images were analyzed using Agilent feature extraction software (version 11.0.1.1; Agilent Technologies, Inc.). The GeneSpring GX v12.1 software package (Agilent Technologies, Inc.) was used to perform quantile normalization and subsequent data processing. Following normalization, mRNAs and lncRNAs, in which at least three of the six samples contained flags in present or marginal ('All Targets Value') were selected for subsequent data analysis.

Gene ontology $(\mathrm{GO})$ and pathway enrichment analysis. GO analysis bonding was used to associate the differentially expressed mRNAs with GO function terms. The threshold for significant GO terms was $\mathrm{P}=0.05$. The $\mathrm{mRNAs}$ were considered to have a higher level of association with the GO term if its P-value was lower. The enrichment pathways of the differentially expressed mRNA were identified using the Kyoto Encyclopedia of Genes and Genomes (KEGG) database with Fisher $\mathrm{P}<0.05$. The GO and KEGG enrichment analyses were performed using the Database for Annotation, Visualization and Integrated Discovery (DAVID; https://david.ncifcrf. gov/).

Establishment of the IncRNA and mRNA co-expression network. The lncRNA-mRNA co-expression pairs were based on the normalized signal intensity of microarray data for the mutant and wild-groups. Pearson's correlation coefficients (PCCs) were used to calculate the correlation between lncRNA and mRNA pairs for all combinations among all the differentially expressed lncRNAs and mRNAs. The pairs with PCC values $\geq 0.95$ or $\leq-0.95$ and with $\mathrm{P}<0.01$ were selected as linkages in the network, and were considered to be significantly correlated pairs. The lncRNA and mRNA co-expression network was constructed using Cytoscape software version 3.3.0 (http://www.cytoscape.org/) (10).

GO BP prediction of the differentially expressed IncRNAs. The GO BPs of the differentially expressed lncRNAs were predicted using a computational method, gene set enrichment analysis (GSEA) (11). This method interprets gene expression data by focusing on gene sets, which share common biological function, chromosomal location or regulation. The expression level of each differentially expressed lncRNA was considered as a profile and correlated with all protein-coding genes of the microarray by computing the PCCs. For each lncRNA, a list of correlation-based ranked protein-coding genes was constructed. In the present study, GSEA was based on the mice GO BP-relevant gene sets. These gene sets were obtained from enrichment mapping (mice_GO_bp_with_GO_ iea_entrezgene.gmt; http://baderlab.org/GeneSets, Accessed February 24, 2015), which covers 13,190 GO BP terms, with corresponding genes representing the gene set for each $\mathrm{GO}$ term. This ranked list was used to calculate an enrichment score, which is a running sum beginning from the highest correlating gene, and the significant gene sets were identified using the weighted Kolmogorov-Smirnov test. To obtain the false discovery rate P-values, the gene sets were permuted 1,000 times to obtain 1,000 random gene sets. Any gene set of GO BP terms with a family-wise error rate $\mathrm{P}<0.05$ was determined to be significantly associated with the corresponding lncRNA, and the GO BP was considered to be a predicted BP of that particular IncRNA.

Statistical analysis. The expression levels of lncRNA and mRNA between the mutant and wild-groups were compared using a two-tailed t-test. Differentially expressed lncRNAs and mRNAs with statistical significance between the two groups were defined as the genes with absolute fold change $\geq 1.5$ and $\mathrm{P}<0.05$. Hierarchical clustering was performed using R software version 3.1.3 (www.r-project.org).

\section{Results}

Overview of IncRNA and $m R N A$ profiles. The microarray evaluated in the present study contained 35,923 lncRNA probes collected from updated databases and literature, and 24,881 mRNA probes based on the public source, CCDS. The expression profiles of the IncRNA and mRNA are shown in Table I. Only $0.74 \%$ of the expressed lncRNAs $(69 / 9,386)$ were significantly differentially expressed, whereas $0.57 \%$ of the expressed mRNAs $(40 / 7,051)$ were significantly differentially expressed in the two groups (Table I).

As shown in Fig. 1, the distribution of the expressed probes in all the chromosomes of the IncRNAs and mRNAs were determined. For the chromosome distribution of mRNA, the ratio of the expressed probes/total probes of each chromosome was similar with a range of $20-30 \%$, with the exception of the $\mathrm{Y}$ chromosome ratio of only $4.6 \%$. The chromosome distribution of the lncRNA profile was similar, with a range of $30-40 \%$.

Differentially expressed lncRNAs in mutant and wild-type mice. According to the lncRNA expression profiles, among the 
Table I. Summary of results of lncRNA and mRNA profile analysis.

\begin{tabular}{lccc}
\hline Probe class & Total & Expression above background $\mathrm{n}(\%)$ & Differentially expressed $\mathrm{n}(\%)$ \\
\hline lncRNAs & 35,923 & $9,386(26.13)$ & $69(0.74)$ \\
mRNAs & 24,881 & $7,051(28.34)$ & $40(0.57)$ \\
Combined & 60,804 & $16,437(27.03)$ & $109(0.66)$
\end{tabular}

lncRNA, long non-coding RNA.

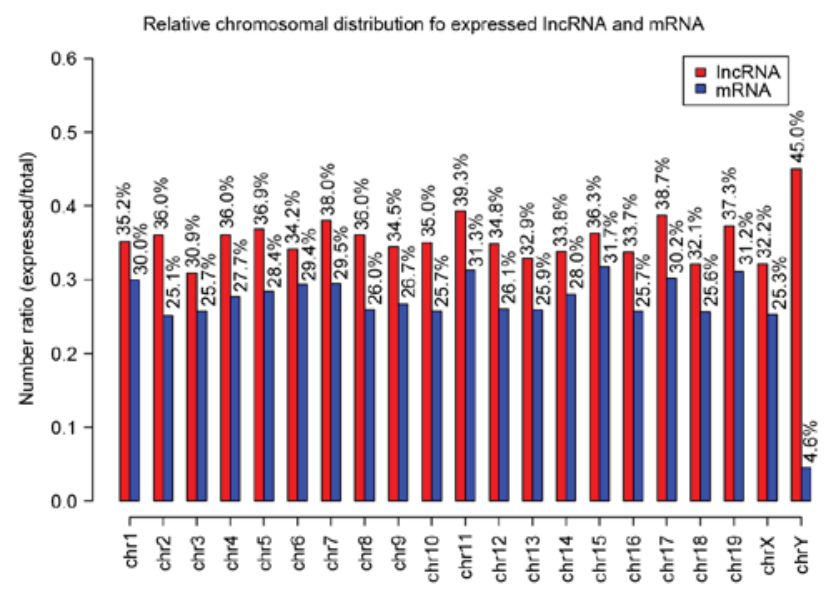

Figure 1. Relative chromosomal distribution of expressed lncRNAs (red) and mRNAs (blue). IncRNA, long non-coding RNA; chr, chromosome.

9,386 probes with expression signals above that of background noise, only 69 lncRNAs were differentially expressed (absolute fold change $>1.5$ and $\mathrm{P}<0.05$ ) between the mutant and wild-mice (Fig. 2A). Among the 69 differentially expressed IncRNAs, 37 lncRNAs were identified as being downregulated and 32 IncRNAs were upregulated with statistical significance in the mutant mice. The unsupervised hierarchical clustering of the 69 differentially expressed lncRNAs for the mutant and wild-types showed that the expression values of each lncRNA in the three samples of the same group were similar, whereas the expression values of each IncRNA in the six samples of the two groups were different (Fig. 2B). The 69 differentially expressed lncRNAs in the two sets of mice samples contained 10 natural antisense (five upregulated and five downregulated), 34 intergenic (17 upregulated and 17 downregulated), eight intronic antisense (four upregulated and four downregulated), two introns (upregulated), 13 exon sense-overlapping (two upregulated and 11 downregulated) and two bidirectional sequences (upregulated), as shown in Fig. 2C.

Differentially expressed mRNAs in mutant and wild-type mice. Among the differentially expressed mRNAs, the number of the upregulated mRNAs (25/40) was almost twice that of the downregulated mRNAs (15/40). The upregulated mRNAs were enriched in several GO terms with statistical significance, with the two most prominent BP terms being placenta development and regulation of system process (Fig. 3A). The downregulated mRNAs were also enriched in several GO terms, including the amine metabolic and glycosaminoglycan biosynthetic processes in BP (Fig. 3B). The analysis performed by mapping genes of the KEGG pathway showed that the upregulated mRNAs were enriched in two KEGG pathways, mRNA surveillance pathways and RNA transport (Fig. 3C), whereas no enriched KEGG pathways were identified for the downregulated mRNAs. These two KEGG pathways contained the two upregulated mRNAs, apoptotic chromatin condensation inducer 1 and nuclear RNA export factor 7.

Determination of the IncRNA and mRNA coexpression network. Coexpression network analysis was performed between the lncRNAs and mRNAs (Fig. 4). The correlation between the 69 differentially expressed lncRNAs and all mRNAs were determined, which revealed 1,670 connections above the threshold, including the lncRNA-lncRNA and IncRNA-mRNA pairs. The network contained 719 nodes with 62 differentially expressed lncRNAs, comprising 32 downregulated and 30 upregulated lncRNAs, and 656 mRNAs involved in the coexpression network. For the 656 mRNAs in the network, there were nine downregulated mRNAs (Bcdin3d, creatine kinase muscle, dopamine receptor D4, enamelin, flavin containing monooxygenase 9, hyaluronan synthase 1 , ring finger protein 128 , spindlin family member 4 and WAS/WASL-interacting protein family member 2) and 11 upregulated mRNAs (4933411K20Rik, butyrylcholinesterase, F-Box and WD repeat domain containing 8, killer cell lectin-like receptor subfamily A, leucine zipper and EF-hand containing transmembrane protein 2, mohawk homeobox, Nxf7, olfactory receptor 1138, PHD finger protein 7, Svop-like and zinc finger protein 451 ) among the 40 differentially expressed mRNAs in the microarray. These deregulated mRNAs were connected with 38 differentially expressed lncRNAs.

Prediction of differentially expressed lncRNA functions through the associated $m R N A$. The analysis of the 69 differentially expressed lncRNAs revealed a set of eight IncRNAs associated with distinct and diverse GO BP terms with statistical significance (ASMM10P055228, ASMM10P040128, ASMM10P056450, ASMM10P040313, ASMM10P044918, ASMM10P039329, ASMM10P024421 and ASMM10P037946). The set of annotated lncRNAs contained six downregulated and two upregulated lncRNAs (Table II).

\section{Discussion}

At present, the OPTN (E50K) mutation of is the only mutation to be identified as one of the origins of NTG 

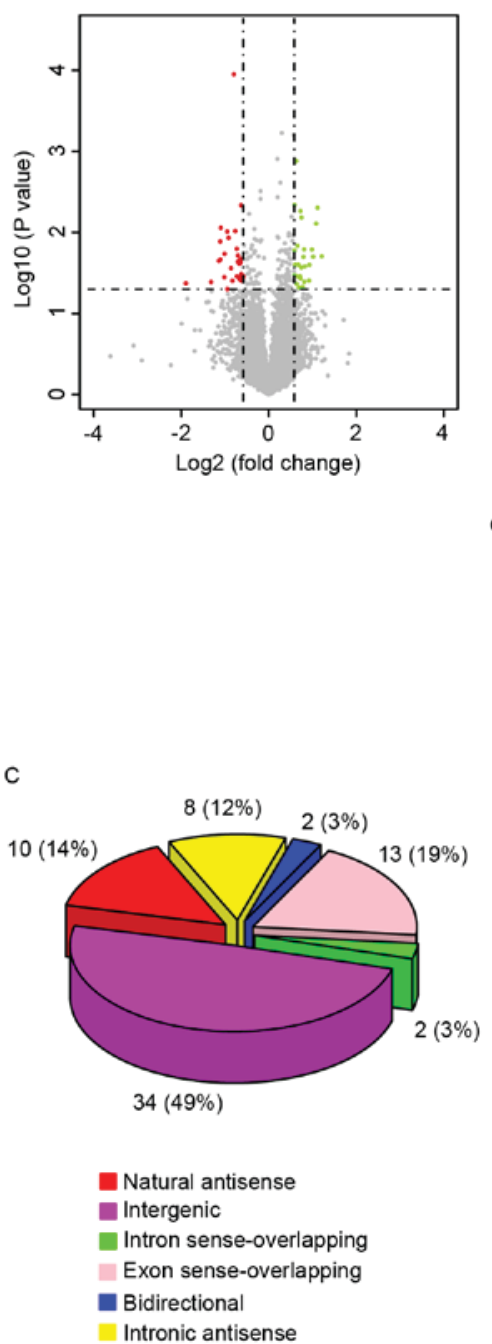
Mutant Wild

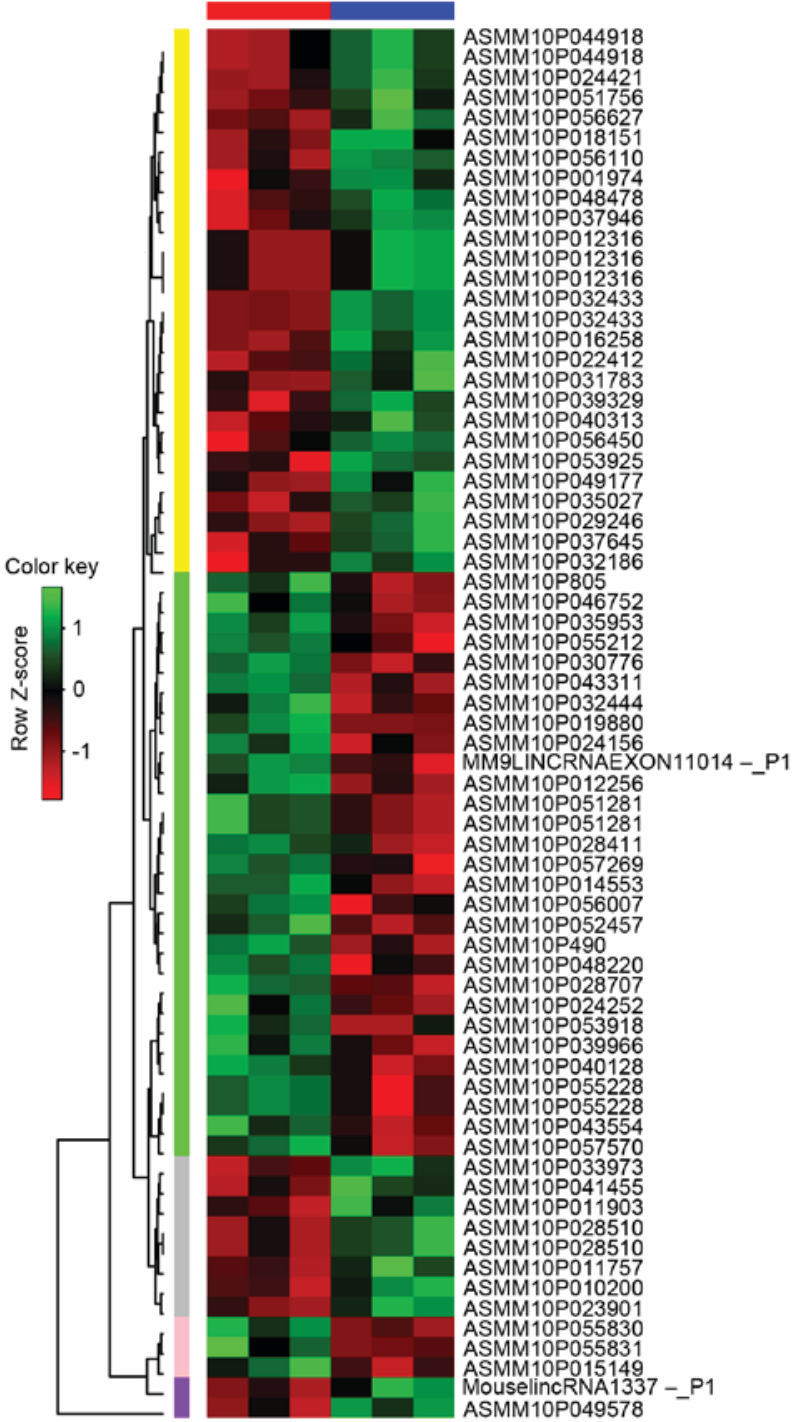

Figure 2. (A) Volcano plot of the identified differentially expressed lncRNAs. (B) Heat map of the differentially expressed lncRNAs identified between mutant and wild-type mice (green, upregulated lncRNAs; red, downregulated lncRNAs). (C) Genomic location of the differentially expressed lncRNAs. lncRNA, long non-coding RNA.

pathogenesis (12). The overexpression of OPTN (E50K) in transgenic mice provides a model system to investigate the molecular mechanisms causing POAG (8). The deregulation of IncRNAs has been associated with the susceptibility to certain human diseases, including cancer, neurological disease, cardiovascular disease and glaucoma (13). However, the role of lncRNAs in OPTN (E50K) transgenic mice has not been investigated. The results of the present study are the first, to the best of our knowledge, to reported the expression profile of lncRNAs in OPTN (E50K) transgenic mice and show that the function of lncRNAs may be novel biomarkers for POAG.

In comparing the expression profiles of 1 ncRNAs and mRNAs, the lncRNAs had a smaller percentage of significantly expressed signals above background expression. However, the number of differentially expressed lncRNAs was higher, compared with the number of differentially expressed mRNAs; and it was revealed that the lncRNAs may perform more important functions in the mutant mice, compared with
mRNAs. It was found that lncRNAs exhibited increased spatially and temporally-regulated expression patterns, compared with protein-coding genes.

In terms of the chromosome distribution of the mRNAs, the ratio of expressed probes/total probes for each chromosome was similar, with a range of 20-30\%, with the exception of the Y chromosome with a ratio of only $4.6 \%$. The chromosome distribution of the IncRNA profile was similar with these findings, with a marginally higher range of $30-40 \%$, with the exception of the $\mathrm{Y}$ chromosome with a higher ratio, compared with that in the mRNA profile. These results suggested that the IncRNAs of the Y chromosome may have had a relatively important role in the mutant mice.

In the present study, prediction of differentially expressed lncRNA functions among the eight annotated lncRNAs, revealed two in subset 1 (ASMM10P055228 and ASMM10P040128), which were annotated with the GO BP, negative regulation of oxidative stress-induced cell death, and regulation of execution phase of apoptosis, respectively 
A

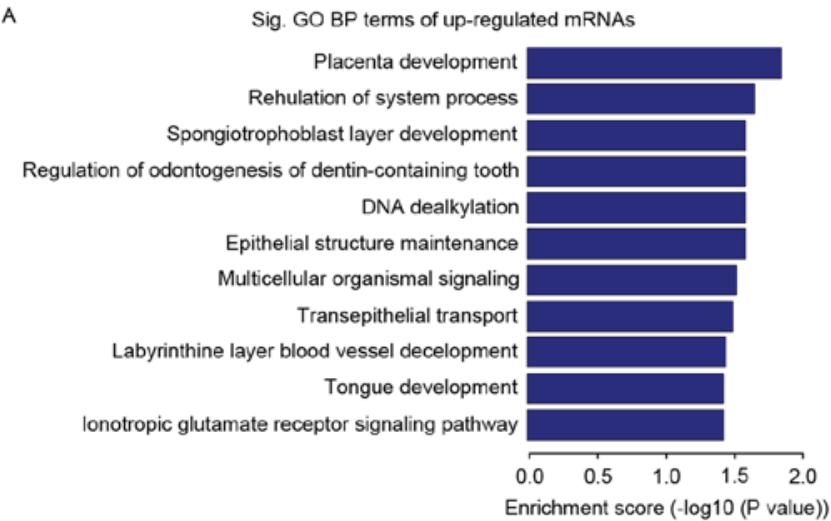

B Sig. GO BP terms of down-regulated mRNAs

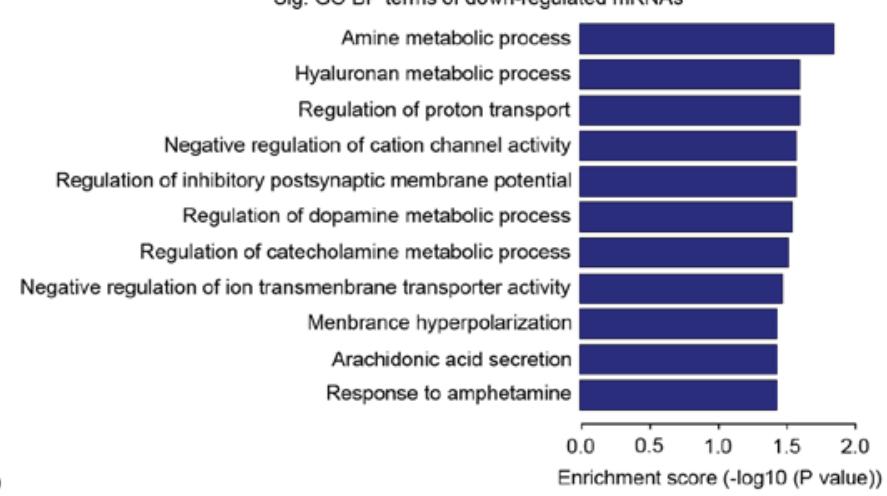

C

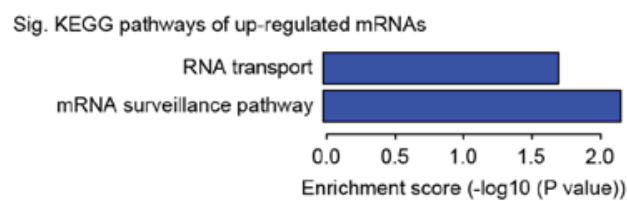

Figure 3. Top 11 GO BP terms of (A) upregulated mRNAs and (B) downregulated mRNAs. (C) Enriched KEGG pathways of the upregulated mRNAs. Sig., significant; GO, Gene Ontology; BP, biological process; KEGG, Kyoto Encyclopedia of Genes and Genomes.

Table II. GO biological process prediction of the differentially expressed long non-coding RNAs by gene set enrichment analysis.

\begin{tabular}{|c|c|c|c|c|c|}
\hline Probe ID & GO name & GO ID & $\begin{array}{l}\text { Enrichment } \\
\text { direction }\end{array}$ & NES & $\begin{array}{l}\text { FWER } \\
\text { P-value }\end{array}$ \\
\hline ASMM10P055228 & $\begin{array}{l}\text { NEGATIVE REGULATION OF OXIDATIVE } \\
\text { STRESS-INDUCED CELL DEATH }\end{array}$ & GO: 1903202 & Negative & -2.019 & 0.017 \\
\hline ASMM10P040128 & $\begin{array}{l}\text { REGULATION OF EXECUTION } \\
\text { PHASE OF APOPTOSIS }\end{array}$ & GO:1900117 & Negative & -1.988 & 0.031 \\
\hline ASMM10P056450 & $\begin{array}{l}\text { PYRIMIDINE-CONTAINING COMPOUND } \\
\text { BIOSYNTHETIC PROCESS }\end{array}$ & GO:0072528 & Positive & 2.019 & 0.044 \\
\hline ASMM10P056450 & $\begin{array}{l}\text { PYRIMIDINE NUCLEOTIDE } \\
\text { BIOSYNTHETIC PROCESS }\end{array}$ & GO:0006221 & Positive & 2.019 & 0.044 \\
\hline ASMM10P040313 & $\begin{array}{l}\text { MYELINATION IN PERIPHERAL } \\
\text { NERVOUS SYSTEM }\end{array}$ & GO:0022011 & Positive & 1.997 & 0.021 \\
\hline ASMM10P040313 & $\begin{array}{l}\text { PERIPHERAL NERVOUS SYSTEM } \\
\text { AXON ENSHEATHMENT }\end{array}$ & GO:0032292 & Positive & 1.997 & 0.021 \\
\hline ASMM10P040313 & $\begin{array}{l}\text { REGULATION OF EXECUTION } \\
\text { PHASE OF APOPTOSIS }\end{array}$ & GO: 1900117 & Positive & 1.983 & 0.032 \\
\hline ASMM10P044918 & $\begin{array}{l}\text { MYELINATION IN PERIPHERAL } \\
\text { NERVOUS SYSTEM }\end{array}$ & GO:0022011 & Positive & 2.053 & 0.010 \\
\hline ASMM10P044918 & $\begin{array}{l}\text { PERIPHERAL NERVOUS SYSTEM } \\
\text { AXON ENSHEATHMENT }\end{array}$ & GO:0032292 & Positive & 2.053 & 0.010 \\
\hline ASMM10P039329 & PROTEIN AUTOUBIQUITINATION & GO:0051865 & Positive & 2.033 & 0.022 \\
\hline ASMM10P039329 & $\begin{array}{l}\text { REGULATION OF DNA-TEMPLATED } \\
\text { TRANSCRIPTION IN RESPONSE TO STRESS }\end{array}$ & GO:0043620 & Positive & 2.029 & 0.022 \\
\hline ASMM10P024421 & $\begin{array}{l}\text { MYELINATION IN PERIPHERAL } \\
\text { NERVOUS SYSTEM }\end{array}$ & GO:0022011 & Positive & 2.115 & 0.007 \\
\hline ASMM10P024421 & $\begin{array}{l}\text { PERIPHERAL NERVOUS SYSTEM } \\
\text { AXON ENSHEATHMENT }\end{array}$ & GO:0032292 & Positive & 2.115 & 0.007 \\
\hline ASMM10P037946 & $\begin{array}{l}\text { PYRIMIDINE-CONTAINING COMPOUND } \\
\text { BIOSYNTHETIC PROCESS }\end{array}$ & GO:0072528 & Positive & 2.025 & 0.037 \\
\hline ASMM10P037946 & $\begin{array}{l}\text { PYRIMIDINE NUCLEOTIDE } \\
\text { BIOSYNTHETIC PROCESS }\end{array}$ & GO:0006221 & Positive & 2.025 & 0.037 \\
\hline
\end{tabular}

GO, Gene ontology; FWER, family-wise error rate; NES, normalized enrichment score. 


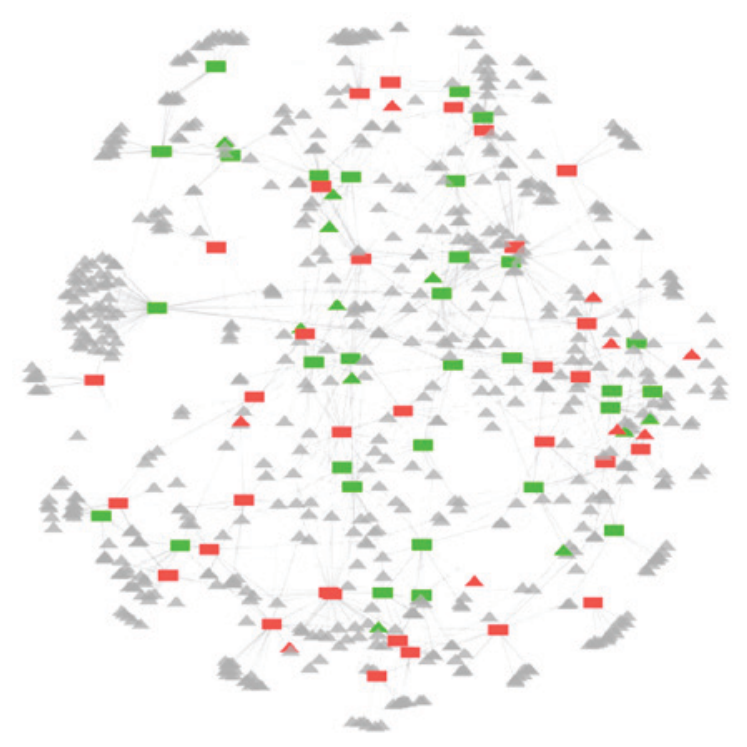

Figure 4. IncRNA and mRNA co-expression network. The rectangular nodes represent the differentially expressed lncRNAs and the triangular nodes represent the mRNAs. The green color represents upregulated IncRNAs or mRNAs, the red color represent downregulated lncRNAs or mRNAs and the grey color indicates mRNAs without expression aberrance. lncRNA, long non-coding RNA.

A

Enrichment plot of ASMM10P055228

GO:1903202 negative regulation of oxidative stress-induced cell death

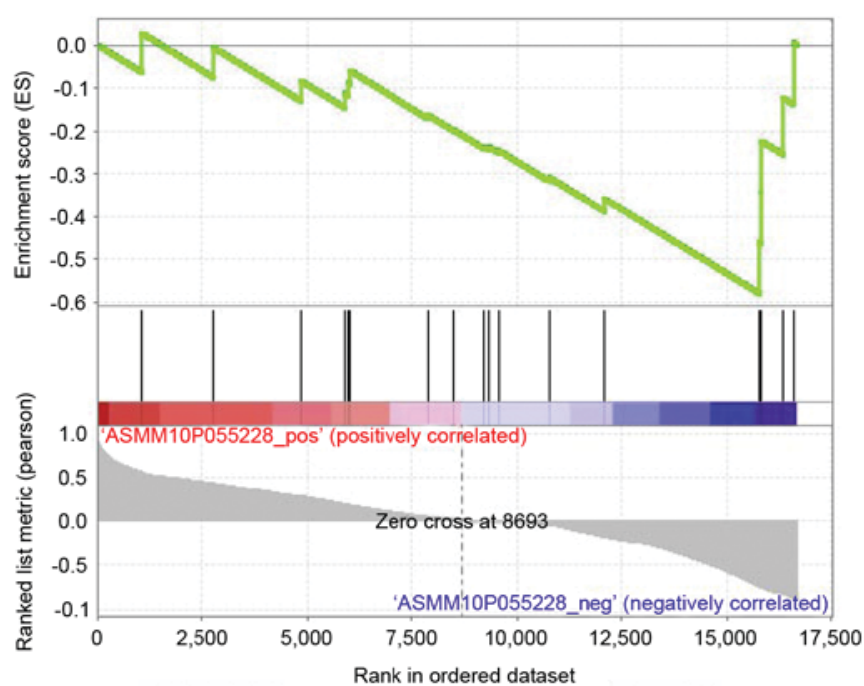

- Enrichment profile - Hits - - Ranking metric scores
B

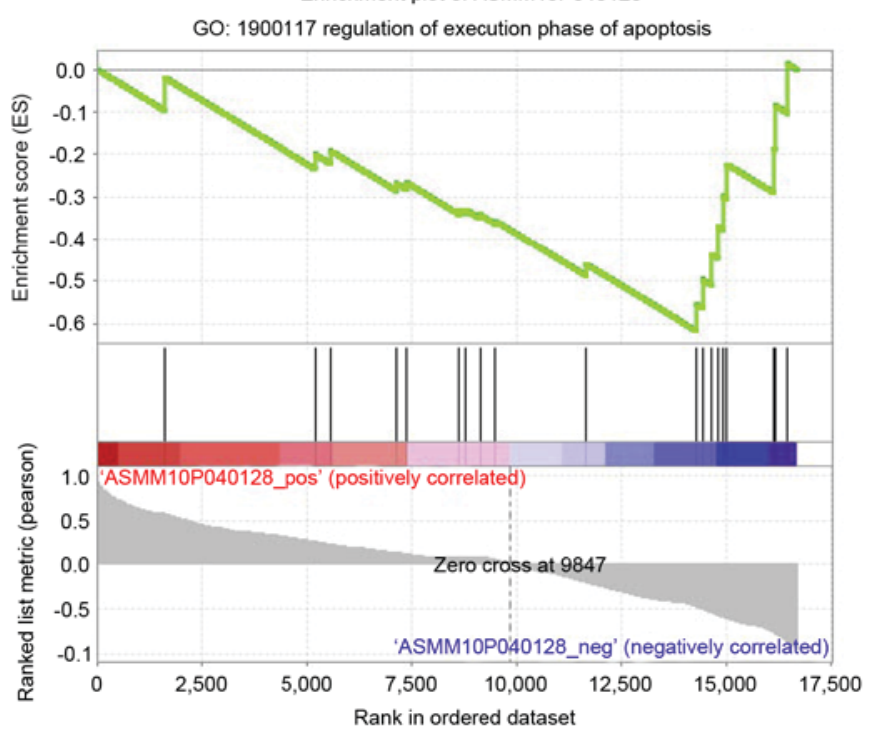

- Enrichment profile - Hits - - Ranking meric scores

Figure 5. GO biological process enrichment of (A) ASMM10P055228 and (B) ASMM10P040128. GO, Gene Ontology.

(Fig. 5A and B). These two GO terms are associated with cell death or apoptosis. The other six lncRNAs were included in subset 2 , and were annotated with seven GO BP terms. Among the six lncRNAs in subset 2, three lncRNAs were annotated with BPs relevant to the nervous system, corresponding with three GO BP terms. The patterns may be involved in similar BPs. Therefore, based on the above, the differentially expressed lncRNAs in the same subset may have the similar biological functions with similar expression patterns. The annotation of ASMM10P055228 and ASMM10P040128 with the negative regulation of oxidative stress-induced cell death and regulation of execution phase of apoptosis, indicate this may be the mechanism underlying POAG.
Investigations are ongoing following those of the present study, and the potential mechanism underlying the observations remains preliminary. Therefore, future investigations are required, focusing on the functional evaluations, to further clarify the pathogenetic molecular pathways, and analyze the effect of different lncRNAs on POAG. The findings of the present study may increase knowledge regarding the function of lncRNAs for detecting the pathogenesis of POAG.

\section{Acknowledgements}

The present study was supported by the State Natural Sciences Foundation (grant nos. 81271000 and 81470634). 


\section{References}

1. Quigley HA and Broman AT: The number of people with glaucoma worldwide in 2010 and 2020. Br J Ophthalmol 90: 262-267, 2006.

2. Fingert JH: Primary open-angle glaucoma genes. Eye (Lond) 25 : 587-595, 2011.

3. Fuse N: Genetic bases for glaucoma. Tohoku J Exp Med 221 $1-10,2010$.

4. Rezaie T, Child A, Hitchings R, Brice G, Miller L, Coca-Prados M, Héon E, Krupin T, Ritch R, Kreutzer D, et al: Adult-onset primary open-angle glaucoma caused by mutations in optineurin. Science 295: 1077-1079, 2002.

5. Aung T, Rezaie T, Okada K, Viswanathan AC, Child AH, Brice G, Bhattacharya SS, Lehmann OJ, Sarfarazi M and Hitchings RA: Clinical features and course of patients with glaucoma with the E50K mutation in the optineurin gene. Invest Ophthalmol Vis Sci: 46: 2816-2822, 2005.

6. Leung YF, Fan BJ, Lam DS, Lee WS, Tam PO, Chua JK, Tham CC, Lai JS, Fan DS and Pang CP: Different optineurin mutation pattern in primary open-angle glaucoma. Invest Ophthalmol Vis Sci: 44: 3880-3884, 2003.

7. Fuse N, Takahashi K, Akiyama H, Nakazawa T, Seimiya M, Kuwahara S and Tamai M: Molecular genetic analysis of optineurin gene for primary open-angle and normal tension glaucoma in the Japanese population. J Glaucoma 13: 299-303, 2004
8. Chi ZL, Akahori M, Obazawa M, Minami M, Noda T, Nakaya N, Tomarev S, Kawase K, Yamamoto T, Noda S, et al: Overexpression of optineurin E50K disrupts Rab8 interaction and leads to a progressive retinal degeneration in mice. Hum Mol Genet 19: 2606-2615, 2010.

9. Ponting CP, Oliver PL and Reik W: Evolution and functions of long non-coding RNAs. Cell 136: 629-641, 2009.

10. Shannon P, Markiel A, Ozier O, Baliga NS, Wang JT, Ramage D, Amin N, Schwikowski B and Ideker T: Cytoscape: A software environment for integrated models of biomolecular interaction networks. Genome Res 13: 2498-2504, 2003.

11. Subramanian A, Tamayo P, Mootha VK, Mukherjee S, Ebert BL, Gillette MA, Paulovich A, Pomeroy SL, Golub TR, Lander ES and Mesirov JP: Gene set enrichment analysis: A knowledge-based approach for interpreting genome-wide expression profiles. Proc Natl Acad Sci USA 102: 15545-15550, 2005.

12. Iwase A, Suzuki Y, Araie M, Yamamoto T, Abe H, Shirato S, Kuwayama Y, Mishima HK, Shimizu H, Tomita G, et al: The prevalence of primary open-angle glaucoma in Japanese: The Tajimi Study. Ophthalmology 111: 1641-1648, 2004.

13. Wapinski $\mathrm{O}$ and Chang HY: Long noncoding RNAs and human disease. Trends Cell Biol 21: 354-361, 2011. 